\title{
An energy balanced wireless sensor network
}

\author{
Lanying $\mathrm{Li}^{\mathrm{a}}$, Weicheng Jiang ${ }^{\mathrm{b}}$, Yong $\mathrm{He}^{\mathrm{c}}$ and Huabing $\mathrm{Li}^{\mathrm{d}}$ \\ The Engineering \& Technical College of Chengdu University of Technology, Leshan Sichuan \\ 614000 , China

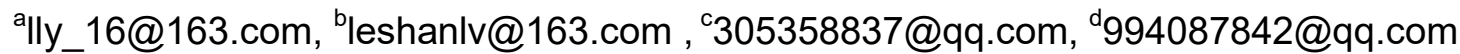

\begin{abstract}
Keywords: Wireless sensor network, Cluster, Energy balance
Abstract: In order to avoid premature death of cluster head nodes, a clustering approach is proposed in this paper. The nodes are labeled as different colors according to the value of the remaining contribution. The cluster head is elected by the color. It is able to reduce the rotation of cluster heads and maintain the stable state of clusters by selecting the nodes with large remaining contributions. In addition, the time as a cluster head is determined by the remaining contribution. It avoids the excessive energy consumption of cluster heads. Results show that the number of death nodes in this algorithm is small. It has a long network lifetime. The correctness of the algorithm is verified.
\end{abstract}

\section{Introduction}

The wireless sensors are arranged in the wild unattended environment, and monitor the specific target [1]. They are often no longer changed once they are arranged. Energy is consumed when sensors are monitoring and transmitting data. After a period of time, many sensors in the network die because of excessive energy consumption. When the number of died sensors reaches a certain amount, the lifetime of the wireless sensor networks will be over. Therefore, energy is a rare resource in wireless sensor networks. It is of great significance to keep the energy efficient and prolong the network lifetime.

It is pointed out that the energy consumption is different in sleep and working state [2]. Try to keep the sensors in sleep, which is good for saving energy. The LEACH algorithm is used to cluster the nodes in the region [3]. The nodes in the cluster transfer the collected data to the cluster head, and the cluster head then transmits the data to the next hop. In order to avoid the excessive energy consumption of cluster heads, the cluster head will be elected after a period of time.

In cluster network, the energy consumption of cluster heads is huge. It is bad for the network if the cluster head died prematurely. In this paper, a method of energy consumption equalization is proposed. Sensors are labeled as different colors, which is the basis for selecting cluster heads.

\section{System design}

In this paper, A parameter is introduced to measure the contribution of wireless sensor nodes to the network in the later stage, called the remaining contribution (indicated as C). Nodes with greater values of $\mathrm{C}$ can make greater contributions to the network in the later stage, and conversely, it will contribute less. The remaining contribution is the basis for nodes to be labeled as different colors. The $\mathrm{C}$ value is determined by two parameters. They are energy (i.e. E) and time (i.e. T). Energy is an important resource in wireless sensor networks. The speed of energy consumption is related to the time. 
Under the same energy, nodes with less work time have great energy and can make great contribution to the network. In addition, under the same working time, nodes with little energy can only make little contribution.

Here is the analysis of the relationship between energy and time. A rectangular coordinate system is established by taking energy as the horizontal axis and the reciprocal of time as the vertical axis. The reciprocal of time is expressed in $\mathrm{V}$. There is $\mathrm{V}=1 / \mathrm{T}$. With the same energy, the nodes with large $\mathrm{V}$ value (less work time) will have greater $\mathrm{C}$, which is represented by longer hypotenuse. With the same $\mathrm{V}$, the nodes with less $\mathrm{E}$ will have smaller $\mathrm{C}$, which is represented by shorter hypotenuse. The length of the hypotenuse can reflect the size of the remaining contribution.

For the same $V$, when $E_{1}$ is less than $E_{2}$, there is $C_{1}<C_{2}$, which can be derived according to the feature of a right triangle as shown in Fig.1. It shows that under the same time, the smaller energy nodes correspond to the smaller remaining contribution.

For the same $\mathrm{E}$, when $\mathrm{V}_{1}$ is less than $\mathrm{V}_{2}$, there is $\mathrm{C}_{1}<\mathrm{C}_{2}$ as shown in Fig.2. It can be derived from the feature of a right triangle. It shows that under the same energy, nodes with smaller $\mathrm{V}$ values correspond to smaller remaining contribution.

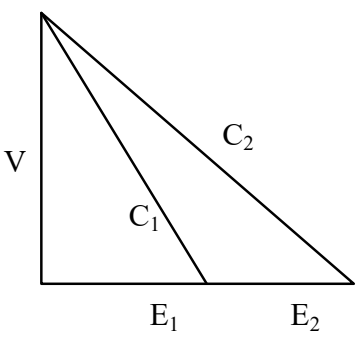

Fig.1. Same V value

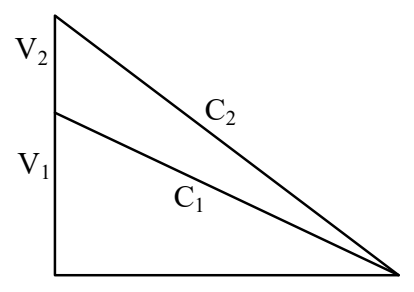

E

The value of the remaining contribution can be calculated according to the formula (1).

$$
C=\sqrt{\alpha_{1} E^{2}+\alpha_{2} V^{2}}=\sqrt{\alpha_{1} E^{2}+\alpha_{2}(1 / T)^{2}}
$$

The $\alpha_{1}$ and $\alpha_{2}$ in the formula (1) are scaling factors. The $\alpha_{1}$ is the proportion of energy.

The $\alpha_{2}$ is the proportion of the $\mathrm{V}$. If the energy consumption is high, the value of $\alpha_{1}$ is slightly great. Otherwise, the value of $\alpha_{2}$ is slightly great. By setting the values of $\alpha_{1}$ and $\alpha_{2}$, time and energy will be adjusted in proportion to the application.

Energy changes at all times. It is related to the state of the sensor. The state of sensor nodes is divided into sleep, working as members, and cluster heads. Time is calculated by the formula (2).

$$
T=K Q=\left(k_{1}, k_{2}, k_{3}\right)\left(\begin{array}{l}
t_{1} \\
t_{2} \\
t_{3}
\end{array}\right)
$$

The sleep time is $t_{1}$. The time of data acquisition and data transmission is $t_{2}$ as a member node. The time as the cluster head is $t_{3}$. The coefficient of proportionality in sleep is $k_{1}$. The coefficient of proportionality in member working is $\mathrm{k}_{2}$. The coefficient of proportionality in cluster head is $\mathrm{k}_{3}$.

In wireless sensor networks, the nodes are divided into different grades according to the size of the remaining contribution, and the nodes are colored to their respective ranks. In different 
application systems, different of rank can be set according to the actual needs. In this paper, four colors are used to label the nodes. Sensors are colored according to the formula (3).

$$
L=\left\{\begin{array}{cc}
R & \left(C<h_{1}\right) \\
Y & \left(h_{1} \leq C<h_{2}\right) \\
G & \left(h_{2} \leq C<h_{3}\right) \\
B & \left(h_{3} \leq C\right)
\end{array}\right.
$$

For nodes whose $\mathrm{C}$ values are less than $\mathrm{h}_{1}$, they are labeled as $\mathrm{R}$. For nodes whose $\mathrm{C}$ values are greater than $h_{1}$ and less than $h_{2}$, they are labeled as $Y$. For nodes whose $C$ values are greater than $h_{2}$ and less than $h_{3}$, they are labeled as $G$. For nodes whose $C$ values are greater than $h_{3}$, they are labeled as B.

The cluster head is selected according to the color of the node when the cluster head rotates. First, nodes labeled as B are chosen as cluster heads because their remaining contributions are largest. Secondly, nodes labeled as G are chosen as cluster heads. Third nodes labeled as Y are selected, and finally nodes labeled as $\mathrm{R}$ are selected. The node that acts as the cluster head will change to another color after working for a period of time, and then cluster head rotation will be performed.

The time span of a cluster head can be calculated according to the formula (1) and the formula (3). The energy of the node is $\mathrm{E}_{\mathrm{t} 3}$. The current color code is $\mathrm{B}$. The color of the cluster head will be changed after time $\mathrm{t}_{3}$.

$$
t_{3}^{\prime}=\frac{1}{k_{3}\left|h_{3}^{2}-\alpha_{1} E_{t 3}^{2}\right|} \sqrt{\alpha_{2}\left|h_{3}^{2}-\alpha_{1} E_{t 3}^{2}\right|}-\frac{1}{k_{3}}\left(k_{1} t_{1}+k_{2} t_{2}\right)
$$

Similarly, the energy of the node is $\mathrm{E}_{\mathrm{t} 2}$. The current color code is $\mathrm{G}$. The color of the cluster head will be changed after time $t_{2}$ '.

$$
t_{2}^{\prime}=\frac{1}{k_{3}\left|h_{2}^{2}-\alpha_{1} E_{t 2}^{2}\right|} \sqrt{\alpha_{2}\left|h_{2}^{2}-\alpha_{1} E_{t 2}^{2}\right|}-\frac{1}{k_{3}}\left(k_{1} t_{1}+k_{2} t_{2}\right)
$$

The energy of the node is $E_{t 1}$. The current color code is $Y$. The color of the cluster head will be changed after time $t_{1}$ '.

$$
t_{1}^{\prime}=\frac{1}{k_{3}\left|h_{1}^{2}-\alpha_{1} E_{t 1}^{2}\right|} \sqrt{\alpha_{2}\left|h_{1}^{2}-\alpha_{1} E_{t 1}^{2}\right|}-\frac{1}{k_{3}}\left(k_{1} t_{1}+k_{2} t_{2}\right)
$$

A timer is set up, which sets time span for a cluster head. The cluster head election is held as soon as the time arrives. It avoids the excessive energy consumption of cluster head. The cluster head do not have to monitor the energy status at all times. It's easier to implement.

In the algorithms, clustering is performed in an inhomogeneous manner. The region with great energy consumption is $R_{1}$, and the area is $S_{1}$, and the density of the sensors is $P_{1}$. The region with little energy consumption is $R_{2}$, and the area is $S_{2}$, and the density of the sensors is $P_{2}$. There are $\mathrm{S}_{1}<\mathrm{S}_{2}$ and $\mathrm{P}_{1}>\mathrm{P}_{2}$. The area with large energy consumption is small and the node density is large, which increase energy density.

This is mainly due to the following factors. The area with great energy consumption is small. The small area makes the collected data less. Less data is processed by cluster heads, and energy consumption is small. High density nodes increase redundant energy. There is more energy in the region for relay data transmission. 
The cluster head transfers the data to the next hop. All the cluster head nodes in the network constitute the transmission and forwarding of the relay data. In order to ensure the normal communication, the following two conditions should be fulfilled when clustering. (1)Two nodes in a cluster are within the effective radius of communication. When a node acts as the cluster head, members can transmit data to the cluster head through less energy. (2)The nodes of the two adjacent clusters are within the effective range of communication. The data can be transmitted between the cluster heads, and the data can be transmitted to the next hop cluster with less energy. The energy model of the formula (4) is introduced in document [4].

$$
E_{T}= \begin{cases}l E_{e}+l \varepsilon_{f s} d^{2} & \left(d<d_{0}\right) \\ l E_{e}+l \varepsilon_{m p} d^{4} & \left(d>d_{0}\right)\end{cases}
$$

It can be seen from the formula (4) that the energy of data transmission will increase dramatically when the communication distance is greater than $d_{0}$. In order to reduce energy consumption, communication radius should be less than $\mathrm{d}_{0}$.

Clusters are divided into cluster head node and member nodes. The cluster head node is responsible for the management of the cluster, the data compression and aggregation of the cluster, and data transmission between clusters. The member nodes are responsible for the data collection in the area, and transmit to the cluster head. When the collected data meets the need, other sensors sleep in order to save energy. They are awakened to work when desired.

\section{Evaluation}

In this paper, Matlab is used to evaluate the performance of the algorithm. The experiments of this algorithm and LEACH algorithm are carried out. RCP is the result of the algorithm in the paper.

The number of death nodes is an important index to evaluate the lifetime of network. The more nodes die in the network, the shorter their effective lifetime is. The number of death nodes is shown in Fig. 3. The number of death nodes in RCP is less than those in LEACH. The death of sensors in RCP is later than those in LEACH. Cluster heads are elected according to the remaining contributions in the proposed algorithm. It is beneficial to the balanced utilization of energy. The sensors are arranged in a non-uniform way. These measures can effectively reduce death nodes.

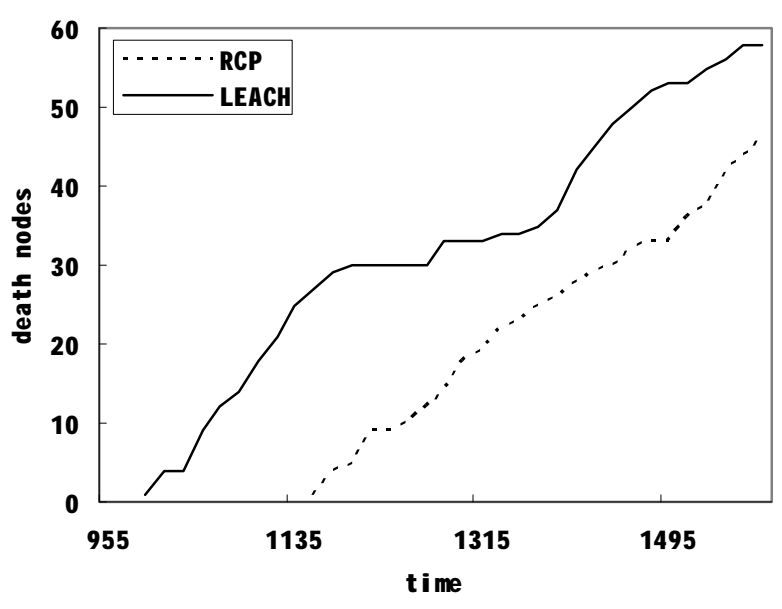

Fig.3. death nodes 


\section{Conclusions}

In the algorithm, sensors are arranged in a non-uniform way. High density sensor nodes are arranged in larger energy consumption areas to increase redundant energy. The sensor nodes are labeled as different colors according to the remaining contribution, which can be used for the election of cluster heads. Nodes those can contribute more to the network are taken as cluster heads. The time span as a cluster head is set according to the value of its remaining contribution. It effectively protects the cluster head from excessive energy consumption. The results show that compared with the LEACH algorithm, the proposed method has longer network lifetime. The correctness of the algorithm is verified.

\section{Acknowledgements}

This work was financially supported by the Education Department of Sichuan Province (16ZB0404) and the Engineering \& Technical College of Chengdu University of Technology (C122015008).

\section{References}

[1] I. F. Akyildiz, W. Su, Y. Sankarasubramaniam, et al. in: A survey on sensor networks. IEEE Communications Magazine, Vol. 40, No. 8, (2002), p. 102

[2] Yang Boxiong, Ni Yuhua. in: Wireless sensor networks. Beijing: People's Posts and Telecommunications Press, (2015), p. 73.

[3] H. Y. Kong. in: Energy efficient cooperative LEACH protocol for wireless sensor networks. Journal of Communications and Networks, Vol. 12, No. 4, (2010), p. 358.

[4] Tian He, J. A. Stankovic, Chenyang Lu, Tarek Abdelzaher. SPEED: A stateless protocol for real-time communication in sensor networks. Proceedings of the 23rd International Conference on Distributed Computing Systems, Washington: IEEE Computer Society, (2003), p. 204. 